\title{
Características de las adolescentes obstétricas. Estudio comparativo con pacientes de 19 a 30 años. Hospital Universitario de Caldas - 1989
}

\author{
Carlos Alberto Esparza Duque*; Mario Calle Mesa**
}

RESUMEN: Comparamos un grupo de 490 primigestantes primíparas adolescentes, de 18 años de edad o menos, con 471 primigestantes primíparas de 19-30 años.

Encontramos dos tipos de diferencias: unas clínica y estadísticamente significativas. Otras sólo clínicamente importantes.

En cuanto a las primeras, está la mayor incidencia de unión inestable y de pacientes sin control prenatal entre las adolescentes. La morbilidad gestacional fue mayor entre las adolescentes en unión inestable $(\mathbf{p}<0.05)$.

Hubo también mayor incidencia de amenaza de parto pretérmino $(p=0.023)$ y de sufrimiento fetal $(p=0.045)$ entre las adolescentes que en el subgrupo testigo de 19-24 años.

En cuanto a diferencias importantes con significancia clínica más no estadística encontramos: mayor incidencia entre las adolescentes, de atonía uterina, sufrimiento fetal intraparto, y parto disfuncional, que en las de 19-24 años y de preeclampsia-eclampsia, amenaza de parto pretérmino, recién nacido pretérmino, bajo peso para la edad gestacional y RCIU que en el grupo de comparación de $25-30$ años.

La incidencia de preeclampsia-eclampsia en adolescentes primigestantes primíparas fue en nuestro estudio de $9.2 \%, 2.2$ veces mayor que el promedio de $4.1 \%$ reportado por Pardo-Uriza (2) en su estudio cooperativo de 11 Instituciones colombianas.

PALABRAS ClaVES: Adolescentes Primigrávidas.

SUMMARY: 490 primigravid primiparous adolescents, 18 years old or less were compared with 471 primigravid primiparous women 19 to 30 years old.

Unstable marital status and lack of prenatal care were found more frequently among adolescents (p<0.05). Morbidity during pregnancy was significantly more frequent among adolescents without stable marital status $(\mathrm{p}<0.05)$.

Other important differences were found between adolescents and control group, namely, uterine atony, fetal intrapartum distress, dysfunctional labor, preeclampsia-eclampsia, threatened preterm delivery, preterm babies, low birth weight for gestational age and IUGP were more frequent among adolescents, but the differences were not statistically significant.

These findings warrant special programs in order to prevent or treat problems detected in primiparous primigravid adolescents.

KEY WORDS: Adolescent Primigravid.

\section{Introducción}

En el estudio anterior presentamos detalladamente las características de 576 adolescentes de 12 a 18 años de edad, atendidas en el Servicio de Obstetricia, del Hospital Universitario de Caldas, entre el 1o. de enero y el 31 de diciembre de 1989.

Lo hicimos intencionalmente así con el propósito de que otros autores que lo deseen puedan comparar sus resultados con los nuestros. Los estudios muy resumidos hacen esta labor prácticamente imposible.

En esta segunda parte, nos proponemos establecer comparación entre el grupo de adolescentes y otro de 471 mujeres de 19 a 30 años de edad cumplidos, y con algunos resultados del estudio cooperativo de Pardo-Uriza, con el

* Profesor Titular. Facultad de Medicina. Universidad de Caldas. ** Profesor Titular. Facultad de Medicina. Universidad de Caldas. fin de precisar si las diferencias justifican o no un manejo especial, del embarazo y del parto, en las adolescentes.

\section{Material y métodos}

Para hacer más homogéneos los grupos decidimos estudiar únicamente primigestantes primíparas. Por este motivo el número de adolescentes se redujo a 490 y el de mujeres de 19 a 30 años que serviá como grupo de comparación, a 471.

Las pacientes se dividieron en 3 subgrupos, de acuerdo con la edad, así:

(1) Adolescentes: 12-18 años. (2) 19-24 años y (3) 2530 años.

El período de estudio es el mismo del trabajo anterior. El registro de los datos y su manejo por computador, se realizaron de la manera descrita allí (1).

Suponemos que las adolescentes obstétricas, en cuanto a su comportamiento durante el embarazo y el 
parto, difieren de los otros dos grupos, en los siguientes aspectos:

1. Morbilidad durante la gestación: infección de vías urinarias, preeclampsia-eclampsia, amenaza de partopretérmino.

2. Morbilidad durante el parto: hemorragia por atonía uterina, sufrimiento fetal agudo, trabajo de parto disfuncional y frecuencia de operación cesárea.

3. Morbilidad del recién nacido: sufrimiento fetal agudo, recién nacido pretérmino, bajo peso para la edad gestacional y RCIU.

Los criterios utilizados para establecer estos diagnósticos fueron los siguientes:

1.1 Infección urinaria: historia, examen físico, parcial de orina, urocultivo con 100.000 o más colonias $/ \mathrm{ml}$.

1.2 Preeclampsia-eclampsia: historia, examen físico, proteinuria parcial de 24 horas, $\mathrm{Hb}$. Hc., recuento de plaquetas, uricemia, examen de fondo de ojo.

1.3 Amenaza de parto pretérmino: historia y examen clínico.

2.1 Hemorragia por atonía uterina: examen clínico.

2.2 Sufrimiento fetal agudo: examen clínico, monitoreo fetal electrónico.

2.3 Trabajo de parto disfuncional: examen físico y partograma siguiendo los criterios de Friedman.

3.1 Sufrimiento fetal por el índice de Apgar al minuto y a los cinco minutos.

3.2 Recién nacido pretérmino, por examen del recién nacido de acuerdo con el método de Capurro.

3.3 Bajo peso para la edad gestacional y RCIU utilizamos el índice de Müller (IM), cuya fórmula es la siguiente:

$\mathrm{IM}=($ Peso en $\mathrm{g} \mathrm{x} \mathrm{100)} /$ (talla) 3

Un IM $=2.35$ a 2.80 corresponde a desnutrido armónico o simétrico.

IM $<2.35$ corresponde a desnutrido disarmónico o asimétrico (RCIU).

Para el análisis estadístico empleamos el cálculo del riesgo relativo (RR), Chi cuadrado y $P$.

\section{Resultados}

1. ESTADO CIVIL

\begin{tabular}{|c|c|c|c|c|c|}
\hline Uniơn & $12-18$ & $19-24$ & $25 \cdot 30$ & P* & $\mathbf{P}^{* * *}$ \\
\hline $\begin{array}{l}\text { Estable } \\
\text { Inestable }\end{array}$ & $\begin{array}{l}220 \\
(44.9 \%) \\
270 \\
(51.1 \%)\end{array}$ & $\begin{array}{l}239 \\
(63.1 \%) \\
140 \\
(36.9 \%)\end{array}$ & $\begin{array}{l}61 \\
(66.3 \%) \\
31 \\
(33.7 \%)\end{array}$ & $0.000+$ & $0.000+$ \\
\hline Total & 490 & 379 & 92 & & \\
\hline
\end{tabular}

* P para comparación de adolescentes con grupo de 19-24

** p para comparación de adolescentes con grupo de 25-30

+ Diferencia estadisticamente significativa

\section{CONTROLES PRENATALES}

\begin{tabular}{|c|c|c|c|c|c|}
\hline Controles & $12-18$ & 19.24 & $25-30$ & $\overline{\mathbf{P}^{*}}$ & $\mathbf{P}^{* * *}$ \\
\hline No. & $\begin{array}{l}89 \\
(18.2 \%)\end{array}$ & $\begin{array}{l}29 \\
(7.7 \%)\end{array}$ & $\begin{array}{l}5 \\
(5.4 \%)\end{array}$ & \multirow[t]{2}{*}{$0.000+$} & \multirow{2}{*}{$0.002+$} \\
\hline Sí & $\begin{array}{l}401 \\
(81.8 \%)\end{array}$ & $\begin{array}{l}350 \\
(92.3 \%)\end{array}$ & $\begin{array}{l}87 \\
(94.6 \%)\end{array}$ & & \\
\hline Total & 490 & 379 & 92 & & \\
\hline
\end{tabular}

* P para comparación de adolescentes con grupo de 19-24

** P para comparación de adolescentes con grupo de 25-30

+ Diferencia estadísticamente significativa

\begin{tabular}{|c|c|c|c|c|c|}
\hline $\begin{array}{r}\text { Edad } \\
\text { Complic. }\end{array}$ & $12-18$ & 19.24 & $25-30$ & $\mathbf{P}^{*}$ & $\mathbf{P}^{* * *}$ \\
\hline $\begin{array}{l}\text { Infección } \\
\text { urinaria }\end{array}$ & $\begin{array}{l}81 \\
(16.5 \%)\end{array}$ & $\begin{array}{l}89 \\
(23.5 \%)\end{array}$ & $\begin{array}{l}15 \\
(16.3 \%)\end{array}$ & 0.336 & 0.957 \\
\hline $\begin{array}{l}\text { Preeclampsi } \\
\text { Eclampsia } \\
\text { Amenaza }\end{array}$ & $\begin{array}{l}45 \\
(9.2 \%)\end{array}$ & $\begin{array}{l}40 \\
(10.6 \%)\end{array}$ & $\begin{array}{l}6 \\
(6.5 \%)\end{array}$ & 0.706 & 0.407 \\
\hline $\begin{array}{l}\text { parto } \\
\text { pretérmino }\end{array}$ & $\begin{array}{l}22 \\
(4.5 \%)\end{array}$ & $\begin{array}{l}9 \\
(2.4 \%)\end{array}$ & $\begin{array}{l}3 \\
(3.3 \%)\end{array}$ & $0.023+$ & 0.422 \\
\hline
\end{tabular}

* P para compa ración de adolescentes con grupo de 19-24

** P para comparación de adolescentes con grupo de 25-30

+ Diferencia estadísticamente significativa

4. COMPLICACIONES DURANTE EL PARTO

\begin{tabular}{|llllll|}
\hline \multicolumn{2}{|l|}{$\begin{array}{l}\text { Edad } \\
\text { Complic. }\end{array}$} & $\mathbf{1 2 - 1 8}$ & $\mathbf{2 5 - 3 0}$ & $\mathbf{P}^{*}$ & $\mathrm{P}^{* *}$ \\
\hline $\begin{array}{l}\text { Atonía uterina } 41 \\
(8.4 \%)\end{array}$ & $\begin{array}{l}30 \\
(7.9 \%)\end{array}$ & $\begin{array}{l}15 \\
(16.3 \%)\end{array}$ & 0.236 & $0.017+$ \\
\hline $\begin{array}{l}\text { Sufrimiento } \\
\text { fetal }\end{array}$ & $\begin{array}{l}27 \\
(5.5 \%)\end{array}$ & $\begin{array}{l}18 \\
(4.7 \%)\end{array}$ & $\begin{array}{l}6 \\
(6.5 \%)\end{array}$ & 0.215 & 0.700 \\
\hline $\begin{array}{l}\text { Parto } \\
\text { disfuncional } 13\end{array}$ & 9 & 4 & 0.441 & 0.274 \\
$(2.7 \%)$ & $(2.4 \%)$ & $(4.3 \%)$ & & \\
\hline
\end{tabular}

* P para comparación de adolescentes con grupo de 19-24

** P para comparación de adolescentes con grupo de 25-30

+ Diferencia estadísticamente significativa

5. CESAREAS

\begin{tabular}{|llllll|}
\hline Edad & $\mathbf{1 2 - 1 8}$ & $\mathbf{1 9 - 2 4}$ & $\mathbf{2 5 - 3 0}$ & $\mathbf{P}^{*}$ & $\mathbf{P}^{* *}$ \\
\hline Cesáreas & 81 & 81 & 29 & 0.069 & $0.000+$ \\
\hline & $(16.5 \%)$ & $(21.4 \%)$ & $(31.5 \%)$ & & \\
\hline
\end{tabular}

* P para comparación de adolescentes con grupo de 19-24

** P para comparación de adolescentes con grupo de 25-30

+ Diferencia estadísticamente significativa

6. COMPLICACIONES DEL RECIEN NACIDO

\begin{tabular}{|c|c|c|c|c|c|}
\hline Complic. & $12 \cdot 18$ & $19-24$ & $25-30$ & $\mathrm{P}^{*}$ & $\mathrm{P}^{* *}$ \\
\hline $\begin{array}{l}\text { Sufrimiento } \\
\text { fetal }\end{array}$ & $\begin{array}{l}46 \\
(9.4 \%)\end{array}$ & $\begin{array}{l}28 \\
(7.4 \%)\end{array}$ & $\begin{array}{l}10 \\
(10.9 \%)\end{array}$ & $0.045+$ & 0.658 \\
\hline $\begin{array}{l}\text { Recién } \\
\text { nacido } \\
\text { pretérmino }\end{array}$ & $\begin{array}{l}33 \\
(6.7 \%)\end{array}$ & $\begin{array}{l}26 \\
(6.9 \%)\end{array}$ & $\begin{array}{l}3 \\
(3.3 \%)\end{array}$ & 0.433 & 0.204 \\
\hline $\begin{array}{l}\text { Bajo peso } \\
\text { para edad } \\
\text { gestacional }\end{array}$ & $\begin{array}{l}27 \\
(5.5 \%)\end{array}$ & $\begin{array}{l}23 \\
(6.1 \%)\end{array}$ & $\begin{array}{l}4 \\
(4.3 \%)\end{array}$ & 0.661 & 0.440 \\
\hline RCIU & 7 & 5 & 1 & 0.608 & 0.631 \\
\hline & $(1.4 \%)$ & $(1.3 \%)$ & $(1.1 \%)$ & & \\
\hline
\end{tabular}

* P para comparación de adolescentes con grupo de 19-24

** P para comparación de adolescentes con grupo de 25-30

+ Diferencia estadísticamente significativa

Comparando adolescentes con mujeres de 19-24 años, observamos diferencias estadísticamente significativas, en los siguientes aspectos:

1. Estado civil: mayor proporción de unión inestable entre las adolescentes.

2. Control prenatal: mayor proporción de adolescentes sin control prenatal.

3. Amenaza de parto pretérmino: mayor en adolescentes.

4. Sufrimiento fetal en la evaluación del recién nacido: mayor en las adolescentes. 
Fue mayor también la proporción de atonía uterina, sufrimiento fetal intraparto y parto disfuncional en las adolescentes, pero las diferencias no fueron estadísticamente significativas. La proporción fue mayor en las pacientes de 19-24 años.

Entre adolescentes y mujeres de 25-30 años, encontramos diferencias estadísticamente significativas, en los siguientes aspectos:

1. Estado civil: mayor proporción de unión inestable en las adolescentes.

2. Control prenatal: mayor proporción de adolescentes sin control prenatal.

3. Hemorragia por atonía uterina: mayor proporción en el grupo de 25-30 años.

4. Frecuencia de cesáreas: mayor frecuencia también en este último grupo.

Hay mayor proporción de preeclampsia-eclampsia, amenaza de parto pretérmino, prematuros, bajo peso para la edad gestacional, y RCIU en el grupo de adolescentes pero estas diferencias no fueron estadísticamente significativas.

\section{Discusión}

El trabajo colaborativo de 11 Instituciones Colombianas, publicado en la Revista Colombiana de Obstetricia y Ginecología, de Abril-Junio de 1991, por Pardo y Uriza (2), coincide con nuestra afirmación previa, en el sentido de que es muy escasa la literatura nacional sobre embarazo y parto en adolescentes: de 1199 referencias sobre adolescencia en general, sólo 25 correspondieron a embarazo en ese grupo etáreo.

La presentación de estos autores, es bastante detallada: ofrece 19 tablas y 24 gráficas. Fácilmente podemos comparar nuestros resultados con los suyos, a diferencia de lo que ocurrió con las publicaciones que citamos en la primera parte de este estudio.

Afirman Pardo y Uriza que el embarazo en adolescentes "es considerado desde el punto de vista obstétrico como un embarazo de alto riesgo por sus múltiples implicaciones sociales y médicas". Citan 5 autores en apoyo de este aserto.

Nuestro estudio se refiere a aspectos médicos y sociodemográficos de embarazo y parto en adolescentes, con el propósito de establecer si tiene "múltiples implicaciones... médicas” y en caso afirmativo, ¿cuáles son en concreto?

La respuesta tiene importancia clínica ya que el 14.9\% de los partos atendidos en el Servicio de Obstetricia del Hospital Universitario de Caldas en 1989, ocurrieron en adolescentes de 18 años o menos. Esta cifra es ligeramente mayor, que la máxima citada por Pardo y Uriza de $14.3 \%$.

Se considera que una unión marital estable brinda apoyo social, económico y psicológico a la embarazada. La unión inestable entre las embarazadas adolescentes fue 1.4 veces más frecuente que entre las del grupo de $19-24$ y 1.5 veces más que en las de 25-30, diferencias éstas altamente significativas. En ellas encontramos mayor morbilidad durante el embarazo y la diferencia fue estadísticamente significativa $(\mathrm{p}<0.05)$.

Las adolescentes sin control prenatal son 2.4 veces más numerosas que las de 19-24 años y 3.4 veces más que las de 25-30.

Podría pensarse que la preeclampsia-eclampsia, considerada por Chesley (3), como típica de primigestantes menores de 25 años, debería ocurrir con frecuencia significativamente mayor en el grupo de adolescentes primigestantes primíparas que en el grupo testigo. Sinembargo no hallamos diferencia significativa con el grupo de 19-24. Pero sí mayor proporción en adolescentes que entre las de 25-30 años, con $\mathrm{p}>0.05$.

En la primera parte de este estudio señalamos que nos parecía baja la incidencia de $7.8 \%$ de preeclampsiaeclampsia en nuestro grupo de adolescentes. Esta proporción es mayor (9.2\%) en el grupo de primigestantes primíparas, objeto del presente informe. En el estudio de Pardo-Uriza, la incidencia de preeclampsia-eclampsia, entre 3252 adolescentes, fue de $4.1 \%$. Nuestra cifra es 2.2 veces mayor y superior al de las 11 instituciones del estudio cooperativo. Sin duda alguna, esta incidencia tiene una gran importancia desde el punto de vista clínico.

Es posible que la diferencia con los datos de PardoUriza, obedezca a los criterios clínicos variables en que se basa el diagnóstico de preeclampsia y también a que nuestro Hospital es un centro de remisión de pacientes de alto riesgo.

El diagnóstico de amenaza de parto pretérmino está rodeado de múltiples factores de incertidumbre. Sinembargo es un diagnóstico que hay que hacer si se quiere modificar la incidencia del gran problema obstétrico del parto prematuro y sus serias consecuencias. La amenaza de parto pretérmino resultó 1.8 veces mayor en las adolescentes que en el grupo de control de 19-30 años y 1.3 veces mayor que en las de 25-30. No encontramos significancia estadística, pero el hecho en sí, es clínicamente importante.

Se puede establecer con mayor seguridad diagnóstico de recién nacido pretérmino. Utilizando este dato tampoco encontramos diferencia estadísticamente significativa. $\mathrm{La}$ incidencia de parto prematuro, por edad gestacional, en el estudio colaborativo de Pardo-Uriza fue de $21.5 \%$ mientras que la nuestra, utilizando el mismo criterio fue de $6.7 \%$ en las adolescentes primigestantes primíparas, es decir, 3.2 veces menor.

Comparando partos espontáneos con partos intervenidos, observamos que estos últimos proporcionalmente son 1.5 veces más frecuentes en el grupo de control que en el de adolescente. La diferencia es estadísticamente significativa. La cesárea es la intervención más frecuente: 1.4 veces más en el grupo de control que en el de adolescentes. Diferencia también estadísticamente significativa. Esto está en contra de lo que espontáneamente tiende a suponerse, en el sentido de que los partos intervenidos son más probables en adolescentes. Además, el porcentaje de cesáreas en estas últimas $(16.5 \%)$ es notablemente menor que el de $23.4 \%$ en el grupo de referencia, hecho clínicamente importante.

Si analizamos las principales indicaciones de las cesáreas en los tres grupos encontramos lo siguiente:

\section{Adolescentes 12-18 años}

- Desproporción cefalopélvica:

- Presentación de pelvis:

$27.2 \%$

- Sufrimiento fetal agudo intraparto: $\quad 22.2 \%$

- Trabajo de parto disfuncional:

- Inducción fallida:

2. Grupo de 19-24 años

- Desproporción cefalopélvica:

$32.1 \%$

- Sufrimiento fetal agudo intraparto:

$24.7 \%$

- Inducción fallida:

- Presentación de pelvis:

- Trabajo de parto disfuncional:

$24.7 \%$

$18.5 \%$

$12.3 \%$

3. Grupo de 25-30 años

- Desproporción cefalopélvica:

- Sufrimiento fetal agudo intraparto:

$48.3 \%$

- Trabajo de parto disfuncional: 
- Inducción fallida:

$17.2 \%$

- Corioamnionitis:

Como puede observarse, en las adolescentes la presentación de pelvis es un factor que reviste particular importancia en la incidencia de cesáreas. Este hecho se explica en parte porque en ellas fue mayor el porcentaje de prematuros (31.8\% vs. $21.1 \%$ en las de $19-24$ años). El trabajo de parto disfuncional también es más frecuente en ellas como indica- ción de cesárea. Estas diferencias no son estadísticamente significativas $(\mathrm{p}>0.05)$.

Pensamos que estos hallazgos justifican atención especial obstétrica en adolescentes, a los importantes problemas señalados.

\section{Agradecimientos}

Al Doctor Eduardo León Jaramillo Velásquez, por su valiosa colaboración en el análisis estadístico.

\section{BIBLIOGRAFIA}

1. Esparza CA., Calle M. Características de las adolescentes obstétricas. Hospital Universitario de Caldas, 1989.

2. Pardo F., Uriza G. Estudio de embarazo en adolescentes en 11 Instituciones colombianas. Rev. Col. Obstet. Ginecol. 1911; 42: 109.
3. Chesley LC. Diagnosis of preeclampsia. Obstet. Gynecol. 1985; 65: 423. 